\title{
Detection of vertical muons with the HAWC water Cherenkov detectors and it's application to gamma/hadron discrimination
}

\author{
Ariel Zúñiga ${ }^{a}$, Alberto Hernández ${ }^{a, b}$, Aldo Miranda ${ }^{c}$, Ana P. Vizcaya ${ }^{a}$, Andrés \\ Sandoval ${ }^{a}$, Jesús Martínez ${ }^{c}$, Rubén Alfaro ${ }^{* a}$, Ernesto Belmont $^{a}$, Hermes León ${ }^{a}$ for \\ the HAWC collaboration ${ }^{d}$ \\ ${ }^{a}$ Instituto de Física, Universidad Nacional Autónoma de México, México D.F, México. \\ ${ }^{b}$ Facultad de Ingeniería, Universidad Autónoma de Querétaro, Querétaro, México. \\ ${ }^{c}$ Centro de Investigación en Computación, Instituto Politécnico Nacional. México D.F, México. \\ ${ }^{d}$ For a complete author list, see www.hawc-observatory.org/collaboration/icrc2017.php \\ E-mail: arzure89@gmail.com
}

\begin{abstract}
The HAWC observatory reconstructs atmospheric showers induced by very high-energy primary cosmic- or gamma-rays. Cosmic rays are several orders of magnitude more frequent than primary gammas and therefore it is necessary to perform the best gamma-hadron discrimination for each event to be able to survey the gamma-ray sky. Single vertical muons cause very characteristic signals in the 300 HAWC water Cherenkov detectors. In particular, those events that produce signals in the four PMTs of one $7.3 \mathrm{~m}$ diameter, $4.5 \mathrm{~m}$ high tanks corresponding to muons traversing the tank in the central region. These events have a correlation that the largest signal is also the earliest to arrive due to the geometry of the Cherenkov light emission and the fact that relativistic muons travel faster than the light in the medium. This pattern is different from that of electrons, positrons and low energy gammas showering in the first meter of water typical of electromagnetic showers. In this work, we explored whether in air-showers it is possible to identify muon signals and whether this can help in the gamma/hadron discrimination of the recorded events.
\end{abstract}

35th International Cosmic Ray Conference - ICRC2017

10-20 July, 2017

Bexco, Busan, Korea

\footnotetext{
* Presenter.
} 


\section{Introduction}

The High-Altitude Water Cherenkov (HAWC) observatory is an instrument located $4100 \mathrm{~m}$ a.s.l (Puebla, Mexico) with the mission of studying gamma-rays up to the very-high-energy (VHE) domain $(\mathrm{E}>100 \mathrm{GeV})$. The detector is an array of 300 Water Cherenkov Detectors (WCD), each one $4.5 \mathrm{~m}$ high, $7.3 \mathrm{~m}$ diameter and a volume containing $200000 \mathrm{~L}$ of purified water. There are four photomultiplier tubes (PMTs) anchored at the tank bottom: a high-quantum efficiency detector or PMT_C (10-inch diameter) centrally located, surrounded by three equally spaced (8-inch diameter) PMTs (A, B, D); all upward facing (Fig. 1) [1].

The operating principle of HAWC is to sample air-shower particles by recording the Cherenkov light produced when they pass through the water. This coherent radiation is emitted into a forward cone (opening angle $\sim 41^{\circ}$ ) that surrounds the direction of motion of the charged particle. Because the Cherenkov cone in water is so large, nearly every charged particle that enters the tank should be observed by at least one of the four PMTs, which converts the light into an electrical signal measurable by the data acquisition system. The output data corresponding to charge and time of the triggered PMTs is later used for the shower's parameters reconstruction (e.g. core position, arrival direction and energy of the primary, event classification: gamma or hadron).

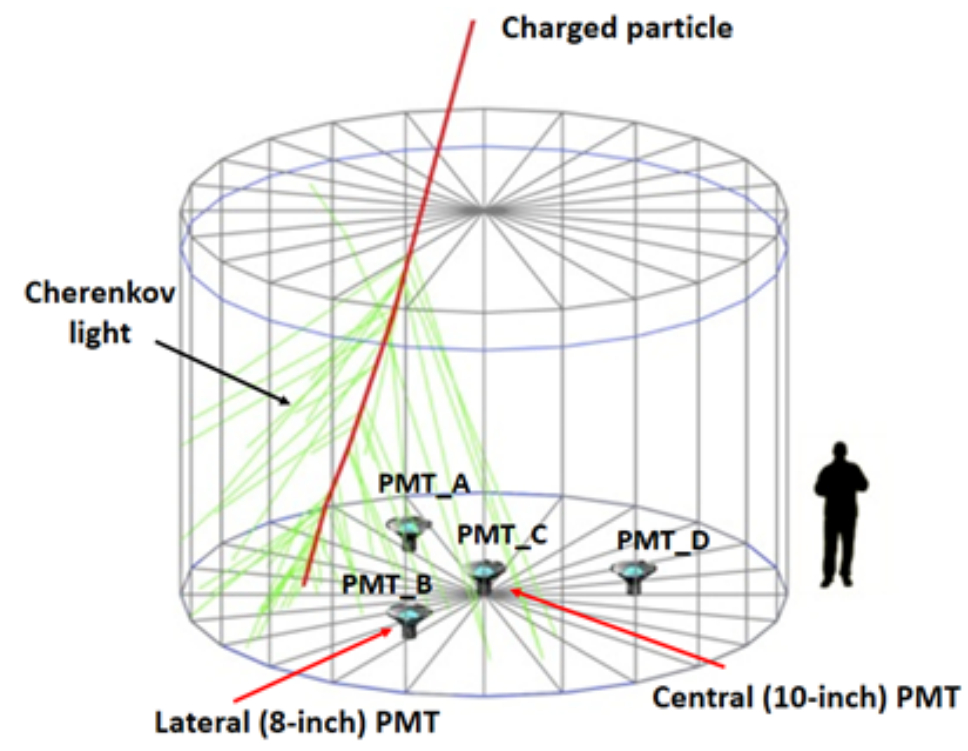

Figure 1: WCD setup, where the passage of a charged particle emitting Cherenkov photons is illustrated.

A common issue of experiments like this is dealing with a large background originated by hadronic primary cosmic rays. Such noise can be thousands of times greater than gamma signals (air-shower trigger rate in HAWC: $\sim 25 \mathrm{kHz}$ ), hence new methods for gamma/hadron separations are needed in order to increase the HAWC's sensitivity to new gamma-ray sources. Motivated by the fact that muon content is quite different in gamma-induced (poor in muons) and hadronicinduced (rich in muons) air-showers ([2],[3],[4]), we study the idea of formulating a new variable for background reduction related with counting the number of muons candidates present in such showers. To reach that goal, we first identified the signature of muons using only time information between photomultiplier tubes. 


\section{Muon time signature in HAWC}

Muons are the most abundant charged particle at the HAWC altitude and arrive mainly with some $\mathrm{GeV}$ of energy. They are originated from the charged meson decays $(\pi, \mathrm{K})$ in air-showers, following approximately straight trajectories through the atmosphere and even inside WCD. Depending on the area where a muon travels it can trigger a specific number of PMTs, this is called multiplicity (M). In this work, we concentrate on vertical muons which travel close to the central light-sensor (PMT_C), the region with the highest probability of having four-multiplicities.

\subsection{Analysis of single muons in raw data}

To choose muon candidates, firstly it was used a sample of raw data. This refers to the processed signal of PMTs before the multiplicity trigger threshold for events selection is applied. We elected all those tanks with four signals $(\mathrm{M}=4)$ within $15 \mathrm{~ns}$ time window. This selection can include electrons $\left(\mathrm{e}^{-}\right)$, positrons $\left(\mathrm{e}^{+}\right)$and low energy gammas $(\gamma)$ which triggered as well all 4PMTs inside a WCD but to help in differentiation we perform Monte Carlo simulations of vertical electrons and muons in a single tank. The energy for particles was: electrons with $1-500 \mathrm{MeV}$ and muons with $15 \mathrm{GeV}$. Then were analyzed the time difference distributions of the PMT_C relative to the peripherals one $\left(t_{A}-t_{C}, t_{B}-t_{C}, t_{D}-t_{C}\right)$. Figure 2 shows that the selected raw data and vertical muon distributions present a similar shape. The mean value (displaced to the right side $=$ time $\mathrm{C}$ often smaller) is approximately $5 \mathrm{~ns}$, a characteristic time in which the laterals PMTs see the Cherenkov light after muons trigger the central detector. However, electron distributions are quite different with a time difference average value near $0 \mathrm{~ns}$, meaning that the PMTs see the light practically at the same time. This happens because electrons stop quickly in the first meter of the tank and then the light travels to the bottom falling promptly over all the detectors. On the other hand, muons reach the bottom triggering the PMT_C first, and later Cherenkov photons arrive at the lateral PMTs.
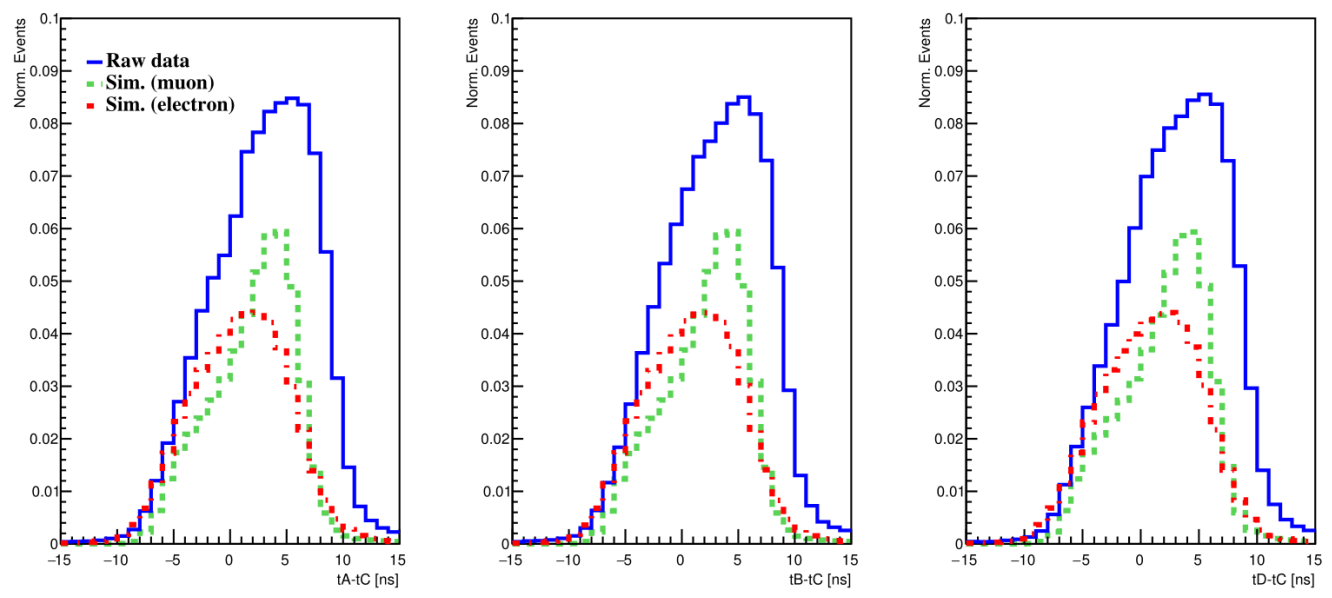

Figure 2: Comparison of the time difference distributions between PMTs for electrons (red), muons (green) and raw data (blue). 


\subsection{Analysis in showers data}

Like gamma showers are mainly electromagnetic (containing a lot of $\mathrm{e}^{-}, \mathrm{e}^{+}$and $\gamma$ ), they must exhibit a time difference close to $0 \mathrm{~ns}$. In order to test this hypothesis, we used to compare simulation data of gamma-induced showers created by the HAWC software and real showers data which passed the trigger condition ( $>28$ PMTs with signal within $150 \mathrm{~ns}$ ).

In the simulation, the energy of gamma primaries range: $5 \mathrm{GeV}-500 \mathrm{TeV}$ and their arrival direction: 0 - 75 degrees from zenith. Such data is generated in extended format, allowing to use the recorded time of each triggered PMT in a shower. Likewise, the information of real cascades was extended data and they are presumably background due to their huge rate. Were set the same restrictions to both kind of data (core inside the HAWC array, all available WCDs with $M=4$, nearby vertical $\left( \pm 10^{\circ}\right)$ showers and time differences with respect to PMT_C). Figure 3 shows time difference distributions produced by showers with unequal muon content. The shower data is rich in muons, this is the reason why the time differences tend to about $5 \mathrm{~ns}$.
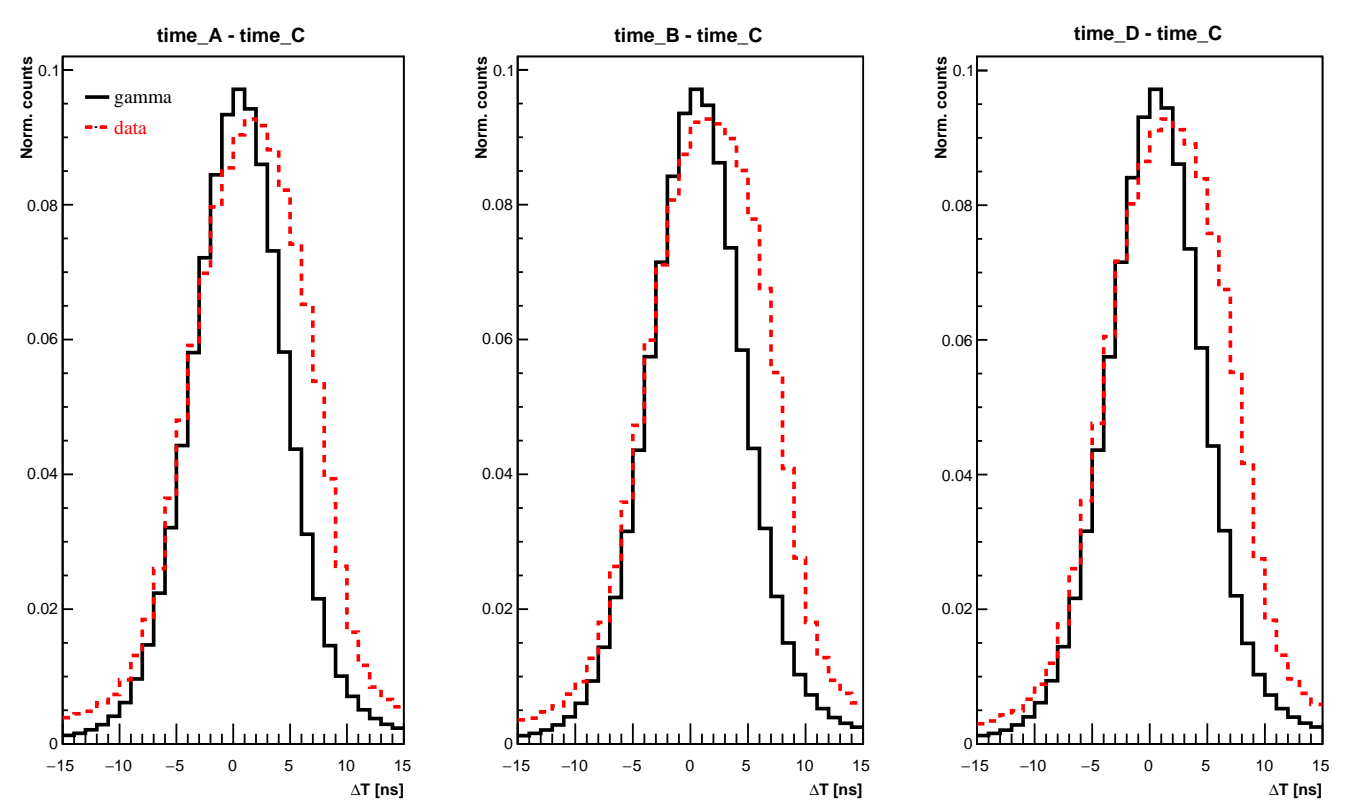

Figure 3: Comparison, using time difference distributions, of showers data (red) and gamma simulated data (black) for tanks with multiplicity 4.

Since the selection of muon candidates is based on identifying the tanks with $M=4$, it is extremely important to have a notion of the frequency they have per event. By means of an algorithm it was determined (Fig. 4). In the previous figure, it is observed that most of the cascades always hit between 45 and 70 tanks. Furthermore, the number of WCDs with $\mathrm{M}=4$ increase with the size of the showers, but the statistics of such events is very low. It is common for cascades of up to 150 tanks to have less than 20 tanks with $\mathrm{M}=4$. 


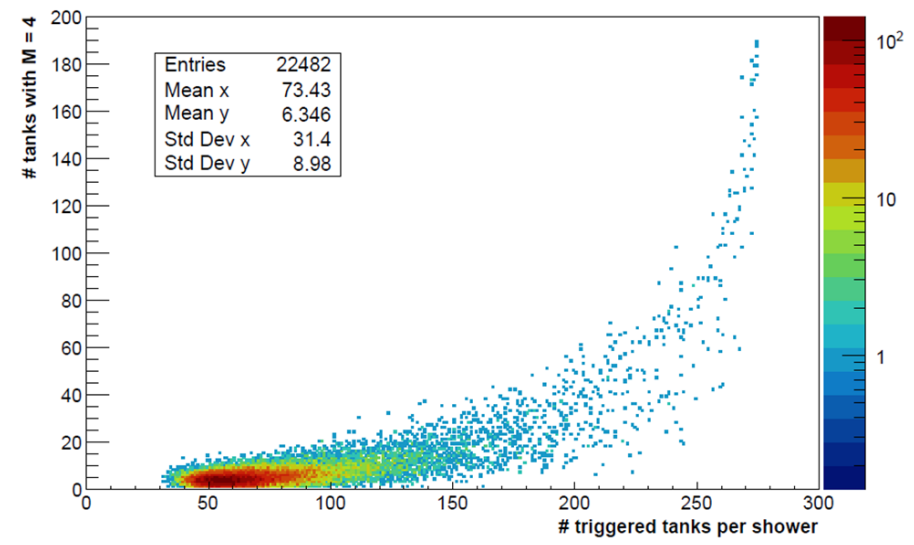

Figure 4: Number of tanks with multiplicity 4 as a function of the shower size.

\section{Definition of a new muon-content-based discrimination parameter}

By making an overlap of the signals with two time differences (Fig. 5 - left), it can be seen that gammas (black dots) have a circular concentration whose center is close to zero, whereas the noise data (red dots) has an elliptical form, spread towards more large values in time difference.

The idea of the variable born out of agglutinating the coincident data within a cutting sphere with a specific radius $\mathbf{R}$ (Fig. 5 - right). All those red dots outside are probably muons. Each WCD with $\mathrm{M}=4$ is a point in the space of the three time differences at a distance $r_{i}$ from origin:

$$
r_{i}=\sqrt{\left(t_{A}-t_{C}\right)^{2}+\left(t_{B}-t_{C}\right)^{2}+\left(t_{D}-t_{C}\right)^{2}}
$$

In Fig. 6 its shown the distribution of $r_{i}$ for simulated gamma and measured hadronic showers. The value of $\mathbf{R}$ was set to $7 \mathrm{~ns}$, such cut maximizes the number of muons out. Therefore, using the restriction $r_{i}>7 \mathrm{~ns}$ were picked out the muon candidates. The new discrimination parameter, named MUDECIDER is based on counting muons per event and the higher values correspond to hadronic-induced showers.
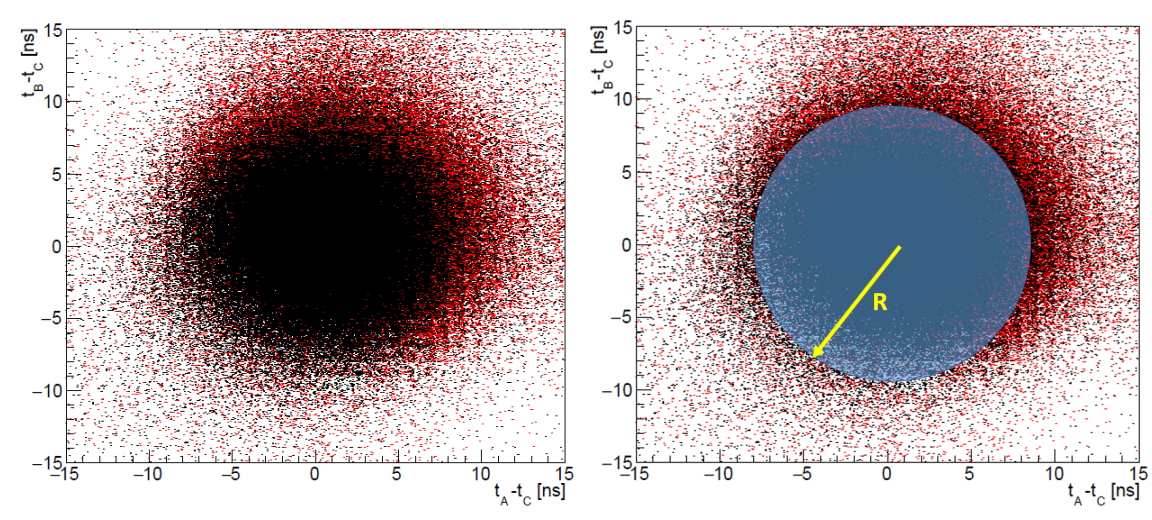

Figure 5: (Left) 2D plot with 2 time differences corresponding to simulated gamma (black points) and real showers data (red points). Missing the third time difference, $t_{D}-t_{C}$. (Right) Region of the cutting sphere with a defined radius $\mathbf{R}$. 


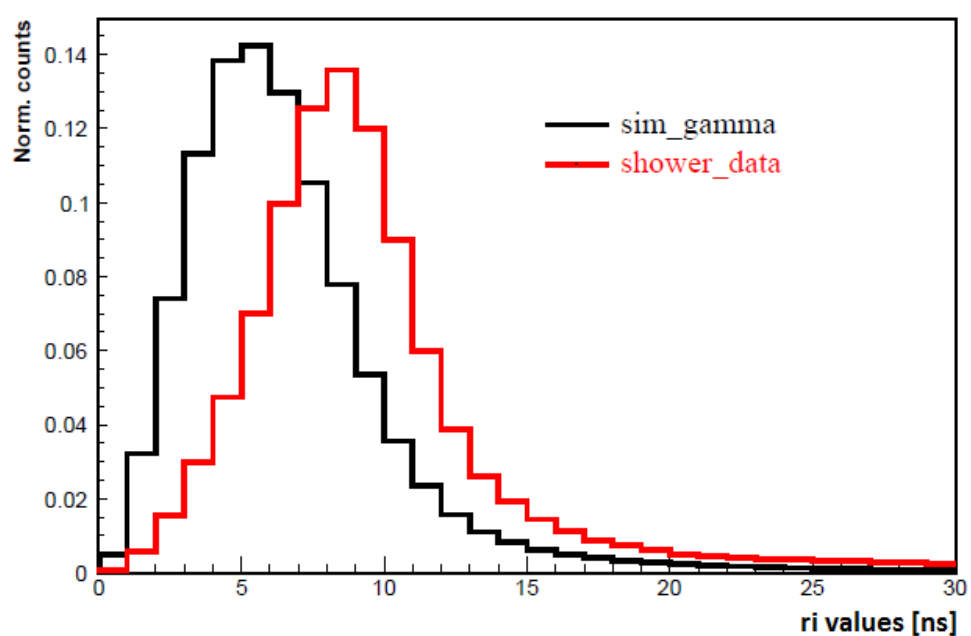

Figure 6: Histogram of $r_{i}$ for both gamma and real-showers data.

Adding a new project to the HAWC software the variable was implemented. Three types of reconstructed data with the output information of MUDECIDER were generated, a normal reconstruction with the minimum threshold established by HAWC ( $>28$ hit PMTs) and two others corresponding to showers where recorded signal more than 300 and 750 PMTs (Fig. 7). The cuts in the number of triggered PMTs were applied with the nHit parameter and give an idea of the variable behavior as a function of larger and energetic events.

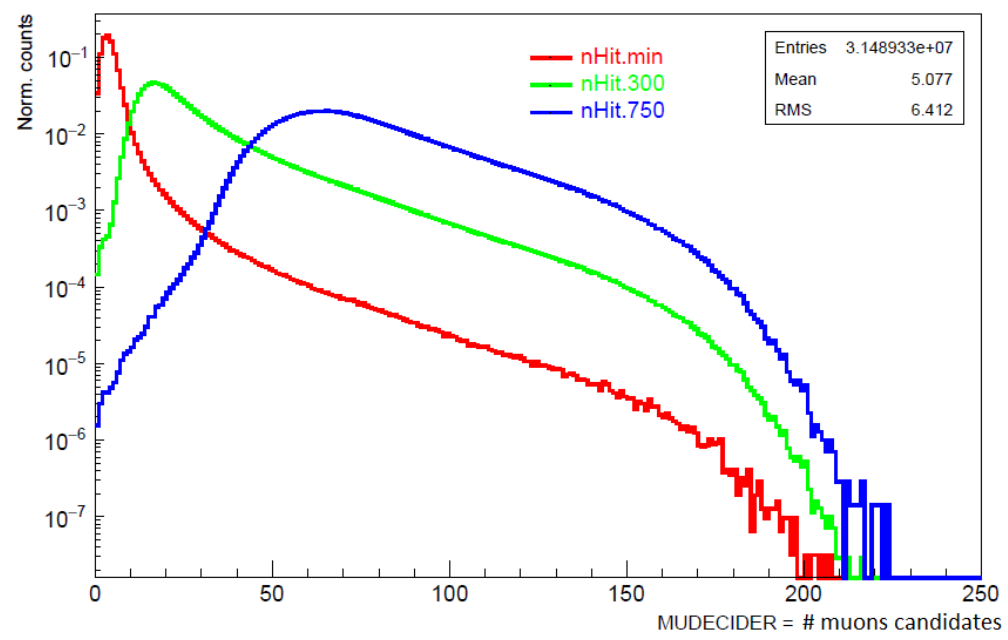

Figure 7: Distributions of MUDECIDER output values as a function of nHit.

It should be noted that increasing nHit more muon candidates are registered, the maximum number is seen between 200 - 230 candidates per shower.

In order to verify MUDECIDER effectiveness, significance maps of the Crab Nebula were compared with and without using it. This astronomical object is the standard candle in $\mathrm{TeV}$ gammaray astronomy and HAWC observes it every day. 
For the analysis in HAWC, cascade events are classified by their size into nine bins [5], depending on the fraction of PMTs registering light in the detector. To visualize the Crab, the second set of reconstructed data was employed (nHit $>300$ ) and correspond to the bins 4-9. A first map (Fig. 8 - left) was created using the official gamma filters, PINCness (Pin) and Compactness (Com); later a second map including MUDECIDER (MU) was built (Fig. 8 - right).

In the normal map (Pin + Com) the maximum significance was $18.53 \sigma$ and the minimum was $-3.63 \sigma$; on the other hand, when the variable ( $\mathrm{MU}+\mathrm{Pin}+\mathrm{Com}$ ) was included, a maximum significance of $19.20 \sigma$ was achieved and the minimum value was $-3.66 \sigma$. In both maps, the maximum was found in the position $\left(83.58^{\circ}, 22.02^{\circ}\right)$ and the minimum in $\left(82.18^{\circ}, 19.63^{\circ}\right)$. The increase in significance was $0.67 \sigma$ and represents approximately $3.5 \%$. The maps have a duration of 555.28 hours and comprise the information of the bins together. The values of MUDECIDER were established per bin analyzing the amount of signal/background in comparison to those produced in the bins of the normal map. The next step is to perform the optimization process of the variable.
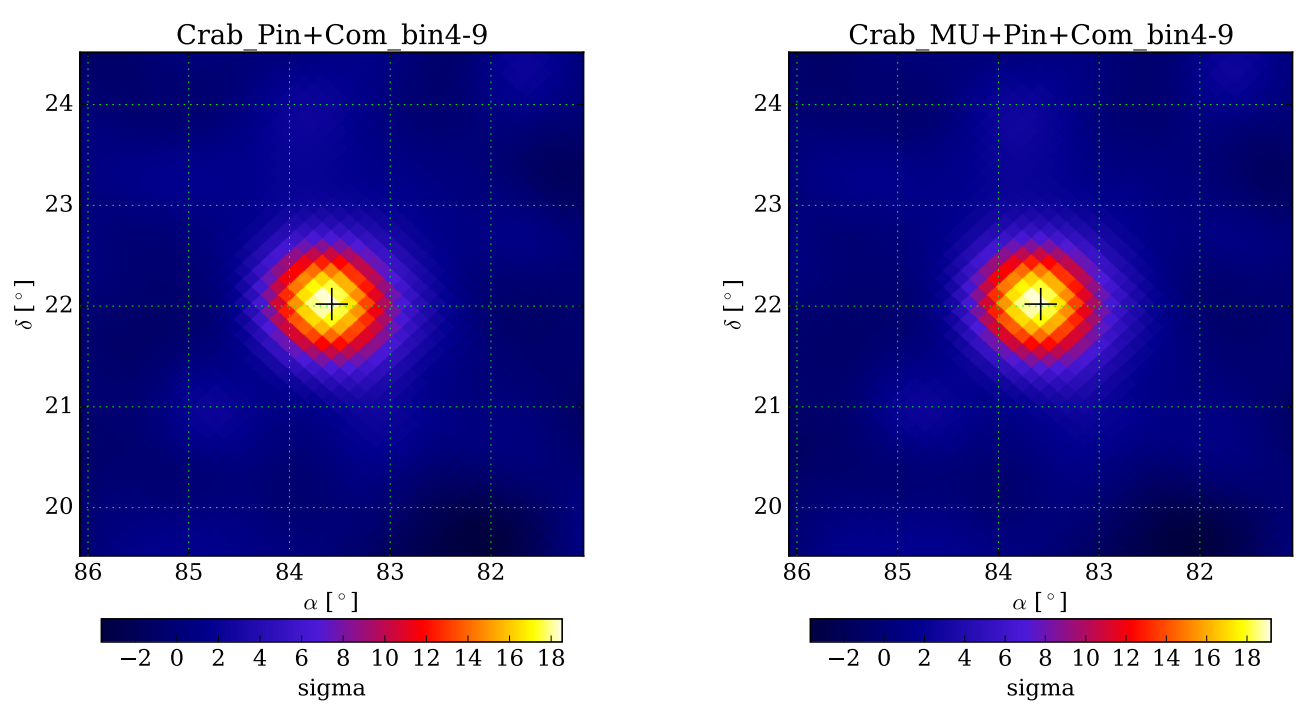

Figure 8: Significance maps of the Crab Nebula without (left) and using (right) MUDECIDER. There is a slight increase in the signal employing the new variable.

\section{Conclusion}

In the presented work, it was analyzed the signature of vertical muons in the HAWC array using only time information. By performing time differences between photomultiplier tubes, we look for muons with trajectories near the central area of tanks which induce multiplicity 4 . Normally, they bring out a characteristic time difference of $\sim 5$ ns between the PMT_C relative to those peripherals, this due to the well-known geometry of the Cherenkov light emission and the arrangement of the PMTs in the WCD. Then it was defined a new (under study) gamma/hadron separation variable based on the muon content of air-showers. Visualizing significance maps of the Crab Nebula, we show promising results of this variable. 


\section{Acknowledgments}

We acknowledge the support from: the US National Science Foundation (NSF); the US Department of Energy Office of High-Energy Physics; the Laboratory Directed Research and Development (LDRD) program of Los Alamos National Laboratory; Consejo Nacional de Ciencia y Tecnología (CONACyT), México (grants 271051, 232656, 260378, 179588, 239762, 254964, 271737, 258865, 243290, 132197), Laboratorio Nacional HAWC de rayos gamma; L'OREAL Fellowship for Women in Science 2014; Red HAWC, México; DGAPA-UNAM (grants IG100317, IN111315, IN111716-3, IA102715, 109916, IA102917); VIEP-BUAP; PIFI 2012, 2013, PROFOCIE 2014, 2015; the University of Wisconsin Alumni Research Foundation; the Institute of Geophysics, Planetary Physics, and Signatures at Los Alamos National Laboratory; Polish Science Centre grant DEC-2014/13/B/ST9/945; Coordinación de la Investigación Científica de la Universidad Michoacana. Thanks to Luciano Díaz and Eduardo Murrieta for technical support.

\section{References}

[1] HAWC Collaboration, A. Smith, HAWC: Design, Operation, Reconstruction and Analysis, in Proc. 34th ICRC, (The Hague, The Netherlands), August, 2015.

[2] P. Grieder. Extensive Air Showers. In: Gamma Ray and Electron Initiated Air Showers (pp. 20). Springer-Verlag Berlin Heidelberg, 2010. eBook ISBN: 978-3-540-76941-5.

[3] M.M. Shapiro, T. Stanev, and J.P. Wefel, editors. Astrophysical Sources of High Energy Particles and Radiation. Springer Science+Business Media Dordrecht, 2001. Volume 44 of Series II: Mathematics, Physics and Chemistry.(pp. 177 - 178)

[4] T. Stanev. Are deep underground detectors good $\gamma$-ray telescopes?, Physical Review D. 33:9, pp. 2740-2743, 1986.

[5] A. Abeysekara et al., Observation of the Crab Nebula with the HAWC Gamma-Ray Observatory, The Astrophysical Journal. 843:39 (17pp), 2017. 